УДК 517.532

\title{
Two-dimensional Motion of Binary Mixture such as Hiemenz in a Flat Layer
}

\author{
Nemat B. Darabi* \\ Institute of Mathematics and Computer Science \\ Siberian Federal University \\ Svobodny, 79, Krasnoyarsk, 660041
}

Russia

Received 10.04.2015, received in revised form 05.05.2015, accepted 08.06.2015

This paper considers solution of thermal diffusion equations in a special type, which describes twodimensional motion of binary mixture in a flat channel. Substituting this solution to equations of motion and heat and mass transfer equations results initial-boundary problems for unknown functions as velocity, pressure, temperature and concentration. If assume that Reynolds number is small (creeping motion), these problems become linear. In addition, they are inverse since unsteady pressure gradient is also desired. Solution of the problem is obtained by using trigonometric Fourier series, which are rapidly convergent for any time value. Exact solution of the stationary and non-stationary problems is presented.

Keywords: thermal diffusion, creeping motion, initial-boundary problem, stationary regime.

DOI: $10.17516 / 1997-1397-2015-8-3-260-272$

\section{Introduction}

Exact and approximate solutions of hydrodynamics equations contribute to a better understanding about qualitative features of motion. They allow us to estimate applicability range of simpler models (non-viscous liquid, creeping motion, boundary layer, and others) and are indispensable for testing numerical, asymptotic and approximate methods. Such solutions are widely used for mathematical modeling of many processes in the chemical and petrochemical technology [1], including convection of mass processes and heat transfer, and various natural phenomena.

This paper deals with unsteady and steady motions of a binary mixture in a flat channel with solid fixed walls. Solution of the thermodiffusion convection equations is sought in a special form: one velocity component is a linear function along the length of channel, and the temperature and concentration are quadratic functions along this coordinate.

First time such solutions for the stationary Navier-Stokes equations are considered by Hiemenz [2]. A review for similar type of exact solutions is available in [3].

In this case, the problem is reduced to a series of one-dimensional inverse problems for parabolic equations (thermal conductivity). For creeping motions (Reynolds number $\operatorname{Re} \ll 1$ ) is found exact solution of stationary and non-stationary problems.

*nematdarabi@gmail.com

(C) Siberian Federal University. All rights reserved 


\section{Problem statement}

Consider a binary mixture. If $C$ is concentration (mass fraction) of component 1 , then concentration of component 2 is $1-C$.

We assume that the pressure gradient causes no significant molecular motion between mixture's components relative to each other, and it is also assumed that deviations of temperature $T$, concentration $C$, and pressure $p$ are small from the average values, and density is a function of $T$ and $C$. So, the mass flux for component 1 can be written as

$$
J=-\rho_{0}\left(D \nabla C+D_{T} \nabla T\right)
$$

where $\rho_{0}$ is mixture density at average temperature and concentration; $D$ is diffusion coefficient; $D_{T}$ is thermal diffusion coefficient. These coefficients, in general, depend on temperature and concentration. However, under the above assumptions, they are constants whose values correspond to average values of temperature and concentration: $D=D\left(T_{a v}, C_{a v}\right), D_{T}=D_{T}\left(T_{a v}, C_{a v}\right)$. The first and second terms on the right hand side of equation (1) describe the flows due to diffusion and thermal diffusion, respectively.

In model Oberbeck-Boussinesq, mixture's motion obeys equation of state

$$
\rho=\rho_{0}\left(1-\beta_{T}\left(T-T_{a v}\right)-\beta_{C}\left(C-C_{a v}\right)\right),
$$

here $\rho_{0}=$ const $>0, \beta_{T}$ is thermal expansion coefficient and $\beta_{C}$ is concentration expansion coefficient.

It is assumed that mixture is located in the gravity field with acceleration vector $\vec{g}=(0,-g)$. Thus motion equations of binary mixture have next form

$$
\begin{gathered}
u_{x}+v_{y}=0 \\
\rho_{0}\left(u_{t}+u u_{x}+v u_{y}\right)+p_{x}=\rho_{0} \mu\left(u_{x x}+u_{y y}\right), \\
\rho_{0}\left(v_{t}+u v_{x}+v v_{y}\right)+p_{y}=\rho_{0} \mu\left(v_{x x}+v_{y y}\right)-g \rho_{0}\left(1-\beta_{T}\left(T-T_{a v}\right)-\beta_{C}\left(C-C_{a v}\right)\right), \\
T_{t}+u T_{x}+v T_{y}=\chi\left(T_{x x}+T_{y y}\right), \\
C_{t}+u C_{x}+v C_{y}=D\left(C_{x x}+C_{y y}\right)+D_{T}\left(T_{x x}+T_{y y}\right) .
\end{gathered}
$$

Using the equality $p_{y}+g \rho_{0}=\partial\left(p+\rho_{0} g y\right) / \partial y=\bar{p}_{y}$ it is possible to rewrite equation (4) as follows

$$
\rho_{0}\left(v_{t}+u v_{x}+v v_{y}\right)+\bar{p}_{y}=\rho_{0} \mu\left(v_{x x}+v_{y y}\right)+\rho_{0} g\left(\beta_{T}\left(T-T_{a v}\right)+\beta_{C}\left(C-C_{a v}\right)\right),
$$

where $u$ is velocity component in direction $x ; v$ is velocity component in direction $y$; $\bar{p}_{y}$ is deviation of total pressure from hydrostatic pressure; $\mu$ and $\chi$ is dynamic viscosity and coefficient thermal diffusivity, respectively.

Fig. 1 shows the scheme of movement field. According to this scheme the boundary conditions arise:

slip on the walls $y=0$ and $y=h$

$$
\begin{array}{ll}
\left.u(x, y, t)\right|_{y=0}=0, & \left.v(x, y, t)\right|_{y=0}=0, \\
\left.u(x, y, t)\right|_{y=h}=0, & \left.v(x, y, t)\right|_{y=h}=0 ;
\end{array}
$$


specified temperatures on these walls

$$
\begin{aligned}
& \left.T(x, y, t)\right|_{y=0}=T_{1}(x, t), \\
& \left.T(x, y, t)\right|_{y=h}=T_{2}(x, t) ;
\end{aligned}
$$

lack of material flow through the walls

$$
D C_{y}+D_{T} T_{y}=0 \quad \text { for } x=0, h .
$$

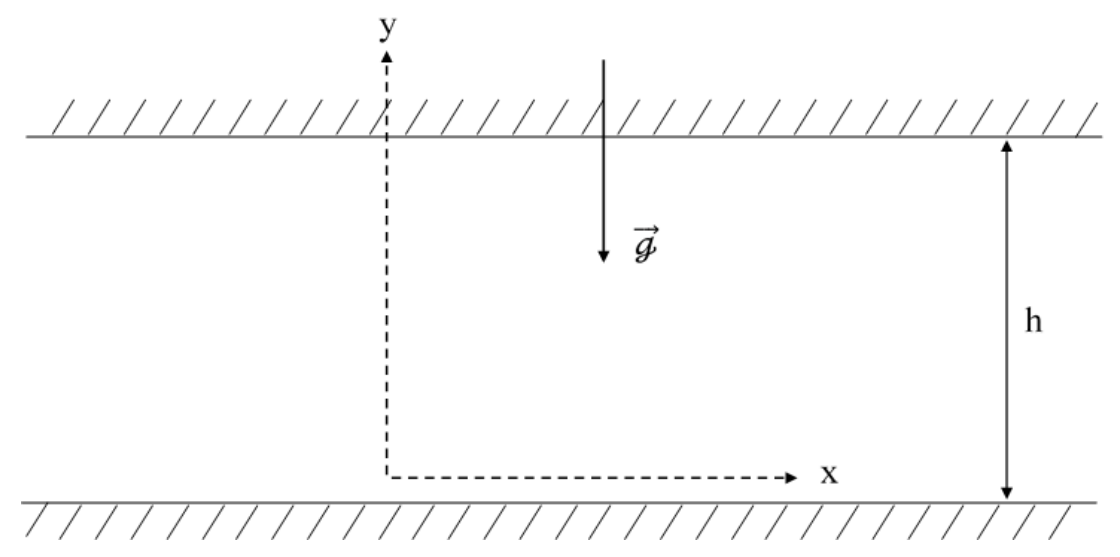

Fig. 1 Scheme of movement field

The initial conditions are

$$
\begin{gathered}
\left.u(x, y, t)\right|_{t=0}=u_{0}(x, y), \\
\left.v(x, y, t)\right|_{t=0}=v_{0}(x, y), \\
\left.u_{x}(x, y, t)\right|_{t=0}=-\left.v_{y}(x, y, t)\right|_{t=0}, \\
\left.T(x, y, t)\right|_{t=0}=T_{0}(x, y), \\
\left.C(x, y, t)\right|_{t=0}=C_{0}(x, y) .
\end{gathered}
$$

In solution of specific problems it is convenient to distinguish characteristic dimensionless quantities (similarity criteria). In connection with defined purpose are used different dimensionless parameters. Here we take dimensionless variables so that in new resulting system of equations as a control dimensionless parameter appeared Reynolds number. To do this, we set

$$
\begin{gathered}
x^{*}=x / h, \quad y^{*}=y / h, \\
u^{*}=u / U_{0}, \quad v^{*}=v / U_{0}, \\
t^{*}=t \mu / \rho_{0} h^{2}, \\
p^{*}=p h / \mu U_{0}, \\
T^{*}=\left(T-T_{a v}\right) / \Delta T, \\
C^{*}=\left(C-C_{a v}\right) \beta_{T} / \beta_{C} \Delta T,
\end{gathered}
$$


where ${ }^{*}$ is shows dimensionless quantities.

It should be noted that in the above formulas, $h$ is channel height is taken as the characteristic of length dimension, $\Delta T$ is characteristic temperature difference in the system, $U_{0}$ is characteristic velocity, $T_{a v}$ and $C_{a v}$ is average temperature and concentration respectively.

Substituting these dimensionless parameters in equations (2)-(6) and also in boundary and initial conditions (7)-(10) and omitting for simplicity upper indices in dimensionless variables, we obtain next equations

$$
\begin{gathered}
u_{x}+v_{y}=0, \\
u_{t}+\operatorname{Re}\left(u u_{x}+v u_{y}\right)+p_{x}=u_{x x}+u_{y y}, \\
v_{t}+\operatorname{Re}\left(u v_{x}+v v_{y}\right)+p_{y}=v_{x x}+v_{y y}+G(T+C), \\
T_{t}+\operatorname{Re}\left(u T_{x}+v T_{y}\right)=\left(T_{x x}+T_{y y}\right) / P r, \\
C_{t}+\operatorname{Re}\left(u C_{x}+v C_{y}\right)=\left(\left(C_{x x}+C_{y y}\right)-\psi\left(T_{x x}+T_{y y}\right)\right) / S c .
\end{gathered}
$$

System (11) contains five dimensionless parameters: Reynolds number $R e=U_{0} h / \mu$, which represents ratio of inertial force to viscous friction force; Prandtl $\operatorname{Pr}=\mu / \chi$ and Schmidt $S c=$ $\chi / D$ numbers, which characterize relative intensity of momentum transfer and molecular heat and mass transfer, respectively; split ratio

$$
\psi=-\beta_{C} D_{T} / \beta_{T} D,
$$

which characterizes effect of thermal diffusion; parameter $G=G r / R e=g \beta_{T} \Delta T h^{2} / U_{0} \mu$ is a measure for relation of buoyancy force to inertial force acting on flow and $G r$ is Grashof number.

Since number of equations (five) is equal to number of unknown functions $(u, v, p, T, C)$, the resulting system (11) will be close one.

Boundary conditions (7)-(9) can be rewritten as

$$
\begin{gathered}
\left.u(x, y, t)\right|_{y=0}=0,\left.\quad v(x, y, t)\right|_{y=0}=0, \\
\left.u(x, y, t)\right|_{y=1}=0,\left.\quad v(x, y, t)\right|_{y=1}=0 ; \\
\left.T(x, y, t)\right|_{y=0}=\left(T_{1}(x, t)-T_{a v}\right) / \Delta T \\
\left.T(x, y, t)\right|_{y=1}=\left(T_{2}(x, t)-T_{a v}\right) / \Delta T ; \\
C_{y}=\psi T_{y} \quad \text { for } x=0,1 .
\end{gathered}
$$

Initial conditions in dimensionless form will take the form

$$
\begin{gathered}
\left.u(x, y, t)\right|_{t=0}=u_{0}(x, y) / U_{0}, \\
\left.v(x, y, t)\right|_{t=0}=v_{0}(x, y) / U_{0}, \\
\left.u_{x}(x, y, t)\right|_{t=0}=-\left.v_{y}(x, y, t)\right|_{t=0}, \\
\left.T(x, y, t)\right|_{t=0}=\left(T_{0}(x, y)-T_{a v}\right) / \Delta T, \\
\left.C(x, y, t)\right|_{t=0}=\beta_{T}\left(C_{0}(x, y)-C_{a v}\right) / \beta_{C} \Delta T .
\end{gathered}
$$

In following section will discuss solution of thermal diffusion equations in a special type, which describes two-dimensional motion in a plane channel. 


\section{Solution in a special kind for initial boundary problems}

We shall seek a solution in the next form

$$
u(x, y, t)=U(y, t) x, \quad v(x, y, t)=V(y, t) .
$$

Applying these representations to motion's equations (11), we find

$$
\begin{gathered}
U(y, t)+V_{y}(y, t)=0, \\
p(x, y, t)=W(y, t) x^{2} / 2+a(y, t), \\
W(y, t)=U_{y y}-U_{t}-\operatorname{Re}\left(V U_{y}+U^{2}\right), \\
T(x, y, t)=A(y, t) x^{2}+B(y, t), \\
C(x, y, t)=M(y, t) x^{2}+N(y, t),
\end{gathered}
$$

where

$$
\begin{gathered}
W_{y}(y, t)=2 G(A(y, t)+M(y, t)), \\
a_{y}(y, t)+V_{t}-V_{y y}+\operatorname{ReV} V_{y}=G(B(y, t)+N(y, t)) .
\end{gathered}
$$

Equations (11) give the system

$$
\begin{gathered}
A_{t}+\operatorname{Re}\left(2 A U+V A_{y}\right)=A_{y y} / \operatorname{Pr} \\
B_{t}+\operatorname{ReV} B_{y}=\left(B_{y y}+2 A\right) / \operatorname{Pr} \\
M_{t}+\operatorname{Re}\left(2 M U+V M_{y}\right)=\left(M_{y y}-\psi A_{y y}\right) / S c \\
N_{t}+\operatorname{ReV} N_{y}=\left(N_{y y}+2 M-2 \psi A-\psi B_{y y}\right) / S c .
\end{gathered}
$$

The boundary conditions (12) reduce to the following

$$
\begin{array}{ll}
\left.U(y, t)\right|_{y=0}=0, & \left.V(y, t)\right|_{y=0}=0 \\
\left.U(y, t)\right|_{y=1}=0, & \left.V(y, t)\right|_{y=1}=0 .
\end{array}
$$

In accordance to (20), (21) and (13) the temperatures on the walls must be of the form

$$
\begin{aligned}
& \left(T_{1}(x, t)-T_{a v}\right) / \Delta T=A_{1}(t) x^{2}+B_{1}(t), \\
& \left(T_{2}(x, t)-T_{a v}\right) / \Delta T=A_{2}(t) x^{2}+B_{2}(t) .
\end{aligned}
$$

With the given functions $A_{j}(t), B_{j}(t), j=1,2$ boundary conditions (14) are converted to four relations

$$
\begin{array}{ll}
\left.M_{y}(y, t)\right|_{y=0}=\left.\psi A_{y}(y, t)\right|_{y=0}, & \left.N_{y}(y, t)\right|_{y=0}=\left.\psi B_{y}(y, t)\right|_{y=0}, \\
\left.M_{y}(y, t)\right|_{y=1}=\left.\psi A_{y}(y, t)\right|_{y=1}, & \left.N_{y}(y, t)\right|_{y=1}=\left.\psi B_{y}(y, t)\right|_{y=1} .
\end{array}
$$

The initial conditions will take following type

$$
U(y, 0)=U_{0}(y), \quad V(y, 0)=V_{0}(y),
$$

furthermore

$$
\begin{gathered}
U_{0}(y)=-V_{0 y}(y) \\
\left(T_{0}(x, y)-T_{a v}\right) / \Delta T=A_{0}(y) x^{2}+B_{0}(y), \\
\beta_{T}\left(C_{0}(x, y)-C_{a v}\right) / \beta_{C} \Delta T=M_{0}(y) x^{2}+N_{0}(y) .
\end{gathered}
$$


Thus, arise the following initial-boundary problems when $0<y<1$ and $t \geqslant 0$ :

$$
\begin{gathered}
A_{t}+R e\left(2 A U+V A_{y}\right)=A_{y y} / \operatorname{Pr} \\
\left.A(y, t)\right|_{t=0}=A_{0}(y) \\
\left.A(y, t)\right|_{y=0}=A_{1}(t) \\
\left.A(y, t)\right|_{y=1}=A_{2}(t) \\
B_{t}+R e V B_{y}=\left(B_{y y}+2 A\right) / P r \\
\left.B(y, t)\right|_{t=0}=B_{0}(y) \\
\left.B(y, t)\right|_{y=0}=B_{1}(t) \\
\left.B(y, t)\right|_{y=1}=B_{2}(t) \\
M_{t}+\operatorname{Re}\left(2 M U+V M_{y}\right)=\left(M_{y y}-\psi A_{y y}\right) / S c \\
\left.M(y, t)\right|_{t=0}=M_{0}(y) \\
\left.M_{y}(y, t)\right|_{y=0}=\left.\psi A_{y}(y, t)\right|_{y=0}, \\
\left.M_{y}(y, t)\right|_{y=1}=\left.\psi A_{y}(y, t)\right|_{y=1} \\
N_{t}+N_{y}=\left(N_{y y}+2 M-2 \psi A-\psi B_{y y}\right) / S c \\
\left.N(y, t)\right|_{t=0}=N_{0}(y) \\
\left.N_{y}(y, t)\right|_{y=0}=\left.\psi B_{y}(y, t)\right|_{y=0} \\
\left.N_{y}(y, t)\right|_{y=1}=\left.\psi B_{y}(y, t)\right|_{y=1} .
\end{gathered}
$$

In following sections discuss stationary and non-stationary solutions of systems (33)-(36) under certain conditions.

\section{Results}

In this section, solutions for both regimes (non-stationary and stationary) are presented.

\subsection{Non-stationary solution}

In systems (33)-(36), it is assumed that $R e \rightarrow 0$ (creeping motion) and $G=G r / R e=O(1)$. The last condition is necessary to account effect of buoyancy force on the motion of mixture. Applying these assumption, will have

$$
\begin{gathered}
A_{t}=A_{y y} / P r, \\
A(y, 0)=A_{0}(y), \\
A(0, t)=A_{1}(t), \\
A(1, t)=A_{2}(t) .
\end{gathered}
$$

By using separation of variables' method we try to solve this system. To achieve this purpose follow the next steps:

$$
D_{k}=2 \int_{0}^{1} \bar{A}_{0}(y) \sin k \pi y d y
$$




$$
f_{k}(t)=2 \int_{0}^{1} f(y, t) \sin k \pi y d y
$$

where

$$
\begin{gathered}
\bar{A}_{0}(y)=\left(A_{1}(0)-A_{2}(0)\right) y+A_{0}(y)-A_{1}(0), \\
f(y, t)=\left(\dot{A}_{1}(t)-\dot{A}_{2}(t)\right)-\dot{A}_{1}(t) ;
\end{gathered}
$$

knowing $D_{k}$ and $f_{k}(t)$ and substituting them we find

$$
g_{k}(t)=e^{-(k \pi)^{2} t / P r}\left(\int_{0}^{t} e^{(k \pi)^{2} \tau / P r} f_{k}(\tau) d \tau+D_{k}\right),
$$

and finally $A(y, t)$ takes next form

$$
A(y, t)=\sum_{k=1}^{\infty} g_{k}(t) \sin k \pi y+\left(A_{2}(t)-A_{1}(t)\right) y+A_{1}(t) .
$$

Equation for $B(y, t)$ after omitting Reynolds number takes the next form

$$
\begin{gathered}
B_{t}=\left(B_{y y}+2 A\right) / P r, \\
B(y, 0)=B_{0}(y), \\
B(0, t)=B_{1}(t), \\
B(1, t)=B_{2}(t) .
\end{gathered}
$$

There is a difference in solution of corresponding equation for $B(y, t)$ compared with solution for $A(y, t)$ and it is in the form of $f(y, t)$ that looks like as

$$
f(y, t)=\left(\dot{B}_{1}(t)-\dot{B}_{2}(t)\right)-\dot{B}_{1}(t)+2 A / P r,
$$

where $A(y, t)$ is found from (37) and the final form for $B(y, t)$ is equal to

$$
B(y, t)=\sum_{k=1}^{\infty} g_{k}(t) \sin k \pi y+\left(B_{2}(t)-B_{1}(t)\right) y+B_{1}(t) .
$$

Note, that for computing $g_{k}(t)$ corresponding boundary and initial values for $B(y, t)$ are used.

Boundary-initial problem for $M(y, t)$

$$
\begin{gathered}
M_{t}=\left(M_{y y}-\psi A_{y y}\right) / S c, \\
M(y, 0)=M_{0}(y), \\
M_{y}(0, t)=\psi A_{y}(0, t), \\
M_{y}(1, t)=\psi A_{y}(1, t) .
\end{gathered}
$$

The first step is to estimate $D_{k}$ and $f(y, t)$ using next relations

$$
\begin{aligned}
D_{k} & =2 \int_{0}^{1} \bar{M}_{0}(y) \cos k \pi y d y, \\
f_{k}(t) & =2 \int_{0}^{1} f(y, t) \cos k \pi y d y,
\end{aligned}
$$


here

$$
\begin{gathered}
\bar{M}_{0}(y)=M_{0}(y)-\psi y^{2}\left(A_{y}(1,0)-A_{y}(0,0)\right) / 2-\psi A_{y}(0,0) y \\
f(y, t)=\psi\left[\left(\left(a_{0}+a_{1} y+a_{2} y^{2}\right) / 2-A_{y y}\right) / S c+\left(A_{y t}(0, t)-A_{y t}(1, t)\right) y^{2} / 2-A_{y t}(0, t) y\right] \\
a_{0}=2 A_{y}(1, t)-2 A_{y}(0, t)+4 A_{y y}(0, t), \\
a_{1}=4 A_{y y}(1, t)-4 A_{y y}(0, t)+2 A_{y y y}(0, t), \\
a_{2}=A_{y y y}(1, t)-A_{y y y}(0, t) .
\end{gathered}
$$

Similar to case for $A(y, t)$ and $B(y, t)$ the next step is finding $g_{k}(t)$

$$
g_{k}(t)=e^{-(k \pi)^{2} t / S c}\left(\int_{0}^{t} e^{(k \pi)^{2} \tau / S c} f_{k}(\tau) d \tau+D_{k}\right),
$$

and at the last step

$$
M(y, t)=\sum_{k=1}^{\infty} g_{k}(t) \cos k \pi y+\psi y^{2}\left(A_{y}(1, t)-A_{y}(0, t)\right) / 2+\psi A_{y}(0, t) y
$$

Solving the problem for $N(y, t)$ completely is similar to one for $M(y, t)$, so we have

$$
\begin{gathered}
N_{t}=\left(N_{y y}+2 M-2 \psi A-\psi B_{y y}\right) / S c, \\
N(y, 0)=N_{0}(y), \\
N_{y}(0, t)=\psi B_{y}(0, t), \\
N_{y}(1, t)=\psi B_{y}(1, t) . \\
D_{k}=2 \int_{0}^{1} \bar{N}_{0}(y) \cos k \pi y d y \\
f_{k}(t)=2 \int_{0}^{1} f(y, t) \cos k \pi y d y
\end{gathered}
$$

where

$$
\begin{gathered}
\bar{N}_{0}(y)=N_{0}(y)-\psi y^{2}\left(B_{y}(1,0)-B_{y}(0,0)\right) / 2-\psi B_{y}(0,0) y \\
f(y, t)=\psi\left(\left(b_{0}+b_{1} y+b_{2} y^{2}\right) / 2-2 A-B_{y y}\right) / S c+ \\
+\psi\left(B_{y t}(0, t)-B_{y t}(1, t)\right) y^{2} / 2-\psi B_{y t}(0, t) y+2 M / S c \\
b_{0}=2 B_{y}(1, t)-2 B_{y}(0, t)+4 B_{y y}(0, t) \\
b_{1}=4 B_{y y}(1, t)-4 B_{y y}(0, t)+2 B_{y y y}(0, t) \\
b_{2}=B_{y y y}(1, t)-B_{y y y}(0, t)
\end{gathered}
$$


therefore $g_{k}(t)$ equal to

$$
g_{k}(t)=e^{-(k \pi)^{2} t / S c}\left(\int_{0}^{t} e^{(k \pi)^{2} \tau / S c} f_{k}(\tau) d \tau+D_{k}\right)
$$

and finally

$$
N(y, t)=\sum_{k=1}^{\infty} g_{k}(t) \cos k \pi y+\psi y^{2}\left(B_{y}(1, t)-B_{y}(0, t)\right) / 2+\psi B_{y}(0, t) y
$$

From equation (20) and (21), knowing $A(y, t), B(y, t), M(y, t)$ and $N(y, t)$ easily are determined temperature and concentration in the mixture.

To find $(u, v, p)$ begin with equation (19) for $\mathrm{U}(\mathrm{y}, \mathrm{t})$, combining equations (19) and (22) for $R e \rightarrow 0$, we obtain

$$
U_{y y}-U_{t}=2 G \int_{0}^{y}(A(z, t)+M(z, t)) d z+w_{0}(t) .
$$

It should be noted that the function $w_{0}(t)$ is also unknown. The function $U(y, t)$ satisfies boundary conditions (28), initial conditions (32) and, in addition

$$
\int_{0}^{1} U(z, t) d z=0
$$

The last follows from slip condition $V(1, t)=0$.

Equation (38) is linear, and it's solution can be sought as the sum of the following two functions,

$$
U(y, t)=U^{1}(y, t)+U^{2}(y, t),
$$

which are solutions of problems

$$
\begin{gathered}
U_{y y}^{1}-U_{t}^{1}=w_{0}(t), \\
U^{1}(0, t)=U^{1}(1, t)=0, \\
U^{1}(y, 0)=U_{0}(y) ; \\
U_{y y}^{2}-U_{t}^{2}=f(y, t), \\
U^{2}(0, t)=U^{2}(1, t)=0, \\
U^{2}(y, 0)=0,
\end{gathered}
$$

where

$$
f(y, t)=2 G \int_{0}^{y}(A(z, t)+M(z, t)) d z
$$

is right-hand side of equation (38) without $w_{0}(t)$.

Solution for $U^{1}(y, t)$ looks like

$$
U^{1}(y, t)=\sum_{k=1}^{\infty} g_{k}(t) \sin k \pi y
$$


with coefficients

$$
\begin{gathered}
g_{k}(t)=g_{k}(0) e^{-(k \pi)^{2} t}-2 q_{k} \int_{0}^{t} e^{-(k \pi)^{2}(t-\tau)} w_{0}(\tau) d \tau, \\
q_{k}=\left(1-(-1)^{k}\right) / k \pi, \\
g_{k}(0)=2 \int_{0}^{1} U_{0}(y) \sin k \pi y d y .
\end{gathered}
$$

In the same way,

$$
U^{2}(y, t)=\sum_{k=1}^{\infty} j_{k}(t) \sin k \pi y
$$

where

$$
\begin{gathered}
j_{k}(t)=-\int_{0}^{t} e^{-(k \pi)^{2}(t-\tau)} f_{k}(\tau) d \tau, \\
f_{k}(t)=2 \int_{0}^{1} f(y, t) \sin k \pi y d y .
\end{gathered}
$$

Consequently $U(y, t)$ is sum of the found functions $U^{1}(y, t)$ and $U^{2}(y, t)$ :

$$
U(y, t)=\sum_{k=1}^{\infty}\left[g_{k}(0) e^{-(k \pi)^{2} t}-\int_{0}^{t} e^{-(k \pi)^{2}(t-\tau)}\left(2 q_{k} w_{0}(\tau)+f_{k}(\tau)\right) d \tau\right] \sin k \pi y .
$$

From the condition (39) we obtain an integral equation for determining $w_{0}(t)$ :

$$
\sum_{k=1}^{\infty} 2 q_{k}^{2} \int_{0}^{t} e^{-(k \pi)^{2}(t-\tau)} w_{0}(\tau) d \tau=h(t)
$$

here

$$
h(t)=\sum_{k=1}^{\infty} q_{k}\left(g_{k}(0) e^{-(k \pi)^{2} t}-\int_{0}^{t} e^{-(k \pi)^{2}(t-\tau)} f_{k}(\tau) d \tau\right) .
$$

Applying Laplace transform $L[r(t)]=\int_{0}^{\infty} e^{-s t} r(t) d t \equiv R(s), \quad[4]$ to both sides of (42), we arrive to equalities

$$
\sum_{k=1}^{\infty} 2 q_{k}^{2} L\left[e^{-(k \pi)^{2} t}\right] L\left[w_{0}(t)\right]=L[h(t)]
$$

whence

$$
w_{0}(t)=L^{-1}\left(\frac{H(s)}{\sum_{k=1}^{\infty} 2 q_{k}^{2} /\left(s+k^{2} \pi^{2}\right)}\right),
$$

where $L^{-1}$ is Laplace inverse. Hence $U(y, t)$ is completely detected from (41), and then using equation $U(y, t)+V_{y}(y, t)=0$ will be found $V(y, t)$. From (23)

$$
a(y, t)=V_{y}-\frac{\partial}{\partial t} \int_{0}^{y} V(z, t) d z+G \int_{0}^{y}(B(z, t)+N(z, t)) d z+a_{0}(t) .
$$

In our problem, without loss of generality, we can set $a_{0}(t) \equiv 0$ and pressure from the equations (18), (19) and (38) is equal to

$$
p(x, y, t)=\left[2 G \int_{0}^{y}(A(z, t)+M(z, t))+w_{0}(t)\right] x^{2} / 2+a(y, t) .
$$

Thus all dimensionless unknown functions $u, v, p, T$ and $C$ are defined. 


\subsection{Stationary solution}

In such case $\partial / \partial t=0$ in equations (11), and denote by $u^{s}, v^{s}, p^{s}, T^{s}$ and $C^{s}$ stationary solution.

Further, using creep motion conditions and $G=G r / R e=O(1)$, we obtain the following linear problem when $0<y<1$ and $t \geqslant 0$ :

$$
\begin{gathered}
A_{y y}^{s}=0, \\
\left.A^{s}(y)\right|_{y=0}=A_{1}, \\
\left.A^{s}(y)\right|_{y=1}=A_{2} ; \\
B_{y y}^{s}=-2 A^{s}(y), \\
\left.B^{s}(y)\right|_{y=0}=B_{1}, \\
\left.B^{s}(y)\right|_{y=1}=B_{2} ; \\
M_{y y}^{s}=0, \\
\left.M_{y}^{s}(y)\right|_{y=0}=\left.\psi A_{y}^{s}(y)\right|_{y=0}, \\
\left.M_{y}^{s}(y)\right|_{y=1}=\left.\psi A_{y}^{s}(y)\right|_{y=1} ; \\
N_{y y}^{s}=2 \psi A^{s}(y)+\psi B_{y y}^{s}-2 M^{s}(y), \\
\left.N_{y}^{s}(y)\right|_{y=0}=\left.\psi B_{y}^{s}(y)\right|_{y=0}, \\
\left.N_{y}^{s}(y)\right|_{y=1}=\left.\psi B_{y}^{s}(y)\right|_{y=1} .
\end{gathered}
$$

Solution of these systems is written by finite formulas. It should be noted that in process of solution (46) (since all the boundary conditions, which are used for defining the desired function are from first order), it is required another condition for determining $N^{s}(y)$. In this task, to overcome this problem, use the following condition

$$
\left.\int_{0}^{1} C(x, y)\right|_{x=0} d y=C_{a v} .
$$

It means that average concentration at $x=0$ is given. With (21) we see that (47) takes the form

$$
\int_{0}^{1} N^{s}(y) d y=C_{a v}
$$

As the case of non-stationary solution after the first stage i.e. finding $A^{s}, B^{s}, M^{s}$ and $N^{s}$ will be searched unknown functions $U^{s}, V^{s}, p^{s}, T^{s}$ and $C^{s}$ but since all of these functions do not depend on the time variable, then using sequential integrations, easily possible to identify them. To achieve this purpose, removing time functions and with equation (38), which connects $A^{s}$ with velocity $U^{s}(y)$, we will have

$$
U_{y y}^{s}=2 G \int_{0}^{y}\left(A^{s}(z)+M^{s}(z)\right) d z .
$$

Next step is to find $V^{s}(y)$ using the equation $U^{s}(y)+V_{y}^{s}(y)=0$, then by following relation, we can find pressure

$$
p^{s}(x, y)=G x^{2} \int_{0}^{y}(A(z)+M(z)) d z+G \int_{0}^{y}(B(z)+N(z)) d z+V_{y} .
$$


In this case, since there isn't any time variable, problems have solved and as a result forms of desire functions are shown.

System of equations (43)-(46) are solved and unknown functions $A^{s}, B^{s}, M^{s}$ and $N^{s}$ look like

$$
\begin{gathered}
A^{s}(y)=\left(A_{2}-A_{1}\right) y+A_{1} \\
B^{s}(y)=y^{3}\left(A_{1}-A_{2}\right) / 3-A_{1} y^{2}+\left(B_{2}-B_{1}-\left(2 A_{1}+A_{2}\right) / 3\right) y+B_{1} \\
M^{s}(y)=\psi\left(A_{2}-A_{1}\right) y+\psi A_{1}=\psi A^{s}(y) \\
N^{s}(y)=\psi B^{s}(y)+\psi\left(7 A_{1}+A_{2}-6 B_{1}-6 B_{2}\right)+C_{a v} .
\end{gathered}
$$

Since in stationary regime all boundary conditions must be constant, so for simplicity in calculations, the values $A_{1}=B_{1}=B_{2}=1, U_{1}=U_{2}=0$ and $A_{2}=-1$ are used. Using these values, we will have

$$
\begin{gathered}
T^{s}(x, y)=(1-2 y) x^{2}+\frac{2}{3} y^{3}-y^{2}-\frac{1}{3} y+1 \\
C^{s}(x, y)=\psi(1-2 y) x^{2}+\psi\left(\frac{2}{3} y^{3}-y^{2}-\frac{1}{3} y+1\right)-6 \psi+C_{a v}, \\
u^{s}(x, y)=\frac{-1}{30}(1+\psi) G x y\left(-1+6 y-10 y^{2}+5 y^{3}\right), \\
v^{s}(y)=\frac{1}{60}(1+\psi) G(-1+y)^{2} y^{2}(-1+2 y), \\
p^{s}(x, y)=\frac{1}{30} G y\left[29+30 C_{a v}-151 \psi+(1+\psi)\left(10 y^{3}-20 y^{2}+y-30 x^{2}(-1+y)\right)\right] .
\end{gathered}
$$

\section{Discussion}

These results derived under two conditions that are noted in Section 3. First condition is associated with creeping movement and second with dimensionless number $G$. These constraints define scale of work and suitable combinations from $h$ and $U_{0}$ for this modeling. As a result it is obtained that, this approach is fair for binary mixtures with big viscosity when $h \sim 10^{-1} \mathrm{~mm}$ and $U_{0} \sim 10^{-1} \mathrm{~mm} / \mathrm{s}$.

In stationary case exact solution is obtained using sequential integrations and it is important to note that it needs additional condition to find all unknown functions.

In process of solving non-stationary mode, method separation of variables is used and solution of the problem derived by using trigonometric Fourier series, which are rapidly convergent for any time value. Accuracy of answers depends on number of sentences, that are considered for series.

\section{References}

[1] A.D.Polyanin, A.M.Kutepov, A.V.Vyazmin, D.A.Kazenin, Hydrodynamics, Mass and Heat Transfer in Chemical Engineering, Topics in Chemical Engineering, vol. 14, Taylor \& Francis, 2001.

[2] K.Hiemenz, The boundary layer on a submerged straight circular cylinder in the uniform liquid flow, Dinglers Polytech Journal, 326(1911), 321-440. 
[3] S.N.Aristov, D.V.Knyazev, A.D.Polyanin, Exact solutions of the Navier-Stokes equations with the linear dependence of velocity components on two space variables, Theoretical Foundations of Chemical Engineering, 5(2009), 547-566 (in Russian).

[4] M.A.Lavrentiev, B.V.Shabat, Methods of Theory of Functions of Complex Variable, Nauka, Moscow, 1973 (in Russian).

\section{Двумерное движение бинарной смеси типа Хименца в плоском слое}

\section{Немат Б. Дараби}

$\overline{B \text { статье рассматривается решение уравнений термодиффузии специального вида, описывающее }}$ двумерное движение в плоском канале. После подстановки этого решения в уравнения движения тепломассопереноса возникают начально-краевые задачи для неизвестных функций скорости, давления, температуры и концентрации. В предположении малости числа Рейнолъдса (ползущие движения) эти задачи становятся линейными. Кроме того, они являются обратными, так как нестационарный градиент давления тоже является искомым. Решение задачи получено с помощъю тригонометрических рядов Фурве, быстро сходящихся для любого момента времени.

Ключевые слова: термодиффузия, ползущее движение, начально-краевая задача, стационарный режим. 